\title{
Design and application of multimegawatt $X$-band deflectors for femtosecond electron beam diagnostics
}

\author{
Valery A. Dolgashev, Gordon Bowden, Yuantao Ding, Paul Emma, Patrick Krejcik,
} James Lewandowski, Cecile Limborg, Michael Litos, Juwen Wang, and Dao Xiang

SLAC National Accelerator Laboratory, Menlo Park, California 94025, USA

(Received 3 December 2013; revised manuscript received 12 August 2014; published 2 October 2014)

\begin{abstract}
Performance of the X-ray free electron laser Linac Coherent Light Source (LCLS) and the Facility for Advanced Accelerator Experimental Tests (FACET) is determined by the properties of their extremely short electron bunches. Multi-GeV electron bunches in both LCLS and FACET are less than 100 fs long. Optimization of beam properties and understanding of free-electron laser operation require electron beam diagnostics with time resolution of about $10 \mathrm{fs}$. We designed, built and commissioned a set of high frequency $X$-band deflectors which can measure the beam longitudinal space charge distribution and slice energy spread to better than $10 \mathrm{fs}$ resolution at full LCLS energy $(14 \mathrm{GeV})$, and with 70 fs resolution at full FACET energy $(20 \mathrm{GeV})$. Use of high frequency and high gradient in these devices allows them to reach unprecedented performance. We report on the physics motivation, design considerations, operational configuration, cold tests, and typical results of the $X$-band deflector systems currently in use at SLAC.
\end{abstract}

DOI: 10.1103/PhysRevSTAB.17.102801

\section{INTRODUCTION}

One of the many technical challenges in LCLS commissioning and operation is the measurement and diagnosis of its extremely short $14 \mathrm{GeV}$ electron bunches. For example, at a low charge of $20 \mathrm{pC}$ the rms bunch length is under $10 \mathrm{fs}$ [1]. It is important that bunch diagnostics include measurements of charge distribution in the phase space along the bunch length (the so-called "beam slice emittance").

In the 1960s SLAC developed technology to measure electron bunch length utilizing a traveling wave structure to produce transverse rf deflecting fields [2,3]. The high frequency time variation of these fields was used to deflect an electron bunch from head to tail by different amounts. When such a beam is transported to a simple profile monitor, the elongated size on the screen represents the bunch length. This is a reliable, single shot measurement of the absolute bunch length.

Since the method effectively converts one of the profile monitor's transverse dimensions into time, the bunch structure can be analyzed in detail. This reveals important time correlations with other phase space dimensions thus allowing the measurement of "time-sliced" energy distribution or other parameters of the beam. This measurement now is an essential diagnostic for the FEL operation of LCLS where such time-sliced beam parameters are of fundamental importance. If the deflector is placed after the

\footnotetext{
*dolgash@slac.stanford.edu
}

Published by the American Physical Society under the terms of the Creative Commons Attribution 3.0 License. Further distribution of this work must maintain attribution to the author $(s)$ and the published article's title, journal citation, and DOI.
PACS numbers: 06.60.Jn, 07.57.-c, 29.20.Ej, 29.27.Fh

undulator, the spent beam can be continuously monitored without interfering with FEL operation. The effects of the undulator on the bunch can be visualized.

The longitudinal resolution of this method $\sigma_{z}$ is given by

$$
\sigma_{z} \gtrsim \frac{\lambda}{\pi e V_{0}} \frac{m_{0} c^{2}}{\sin \Delta \psi} \sqrt{\frac{\gamma \varepsilon_{n}}{\beta_{d}}},
$$

where $\lambda$ is rf wavelength, $m_{0}$ is the electron rest mass, $c$ is the speed of light, $\gamma$ is the relativistic factor of the beam, $\varepsilon_{n}$ is the normalized emittance of the beam, and $e$ is the electron charge. $V_{0}$ is the peak deflecting voltage at crest phase, $\sin \Delta \psi$ is the betatron phase advance from deflector to screen, and $\beta_{d}$ is the beta function at the deflector. The assumption here is that the beam size on the screen doubles with application of the defecting voltage. For details on the derivation see $[4,5]$.

The $3 \mathrm{GHz}$ deflecting structures built and tested for the SLAC linac in the 1960s are now used as indispensable diagnostics for LCLS operation $[1,6]$. An example of such a deflector is LOLA-IV with $2.44 \mathrm{~m}$ length and maximum deflecting voltage of $19 \mathrm{MV}$ at $20 \mathrm{MW}$ input power. We note that one such deflector is insufficient to resolve the beam structure at full LCLS energy. If we wanted to use these $3 \mathrm{GHz}$ LOLA-IV structures downstream of the LCLS undulator to measure bunch structure to about $10 \mathrm{fs}$ resolution, we would need a maximum transverse kick of up to $132 \mathrm{MeV} / \mathrm{c}$. This kick would require seven LOLA-IV structures with a total length of $17 \mathrm{~m}$ and total input power of $140 \mathrm{MW}$ which is not practical. Shortening the deflector $\mathrm{rf}$ wavelength increases its time-resolving power. Two 1-m-long $X$-band deflectors powered by one SLAC XL-4 50 MW klystron provide an equivalent resolution and occupy much less space along the beam line. 
The requirements for such an $X$-band deflector were formulated in [7] which is based on analysis of $S$-band deflector applications to LCLS [4]. The preliminary electrical design of a 1-m-long traveling wave deflector that satisfies these requirements is presented in [8]. The structure described in [8] uses a power coupler never before considered for rf deflectors in order to reduce multipole components of the field [9] and improve high-power performance.

The electrical design of the $X$-band deflector is described in [10]. To design the deflector we applied our experience gained in developing and testing numerous $X$-band high gradient accelerating structures built for the Next Liner Collider project [11,12] and the US High Gradient Collaboration [13].

Existing $X$-band and Ku-band deflectors [14] are used in various applications. Our deflector differs from those because it was developed to satisfy the extreme requirements for femtosecond diagnostics of multi-GeV beams. It uses a traveling wave structure similar to the Radiabeam $X$-band deflector designed to measure a $100 \mathrm{MeV}$ beam [15-17]. Our $X$-band deflector is longer and has a new type of coupler. It is designed to produce a significantly larger transverse kick than compact low power standing wave rf deflectors for femtosecond diagnostics of $\sim 15 \mathrm{MeV}$ beams. Examples of such standing wave structures are $17 \mathrm{GHz}$ deflectors made by Haimson Research Corporation $[18,19]$, and the $9.6 \mathrm{GHz}$ cavity made at UCLA for its Neptune Laboratory [20].

We note that traveling wave $X$-band deflectors are being developed for beam manipulation at the final focus of the Compact Linear Collider (CLIC) [21]. Requirements for that deflector are different from ours as follows: total kick is smaller; multibunch wakefields have to be suppressed; and nondeflecting fields in couplers have to be minimized. To satisfy the last requirement a new type of coupler was recently introduced for the CLIC deflector [9].

At this moment two 1-m-long SLAC $X$-band deflectors are operational at LCLS [22]; one 1-m-long deflector is at FACET [23]; two shorter ones are used at SLAC's Next Linear Collider Test Accelerator (NLCTA) for the Echo experiment [24,25]; and another short one is used for $X$-band gun diagnostics at SLAC's $X$-band Test Facility [26]. In addition to these operational deflectors, one 1-m-long deflector has been sent to the High Energy Research Organization in Tsukuba, Japan (KEK), for use at their B-factory.

This paper is organized as follows: motivation and design requirements leading to the original LCLS deflector (Sec. II); design considerations and design description (Sec. III); manufacturing considerations and selected cold-test data (Sec. IV); practical applications at SLAC and some measurement results (Sec. V).

\section{MOTIVATION AND DESIGN REQUIREMENTS}

The $X$-band deflector design is based on LCLS deflector specifications given in [7], and an earlier $S$-band deflector
TABLE I. Parameter requirements for 10-fs temporal resolution using an $X$-band rf deflecting cavity (Table 1 from [7]).

\begin{tabular}{lcc}
\hline \hline Parameter & Value & Unit \\
\hline Electron energy & 13.6 & $\mathrm{GeV}$ \\
$\begin{array}{l}\text { Desired temporal resolution } \\
\text { Kick amplitude (at } t=\delta t \text { ) in units } \\
\quad \text { of rms beam size }\end{array}$ & 10 & $\mathrm{fs}$ \\
$\begin{array}{l}\text { Radio-frequency wavelength } \\
\quad \text { of deflector }(X \text {-band) }\end{array}$ & 26 & $\mathrm{~mm}$ \\
$\begin{array}{l}\text { Vertical normalized rms emittance } \\
\text { Vertical beta function at the center }\end{array}$ & 1 & $\mu \mathrm{m}$ \\
$\quad$ of the rf deflector & 50 & $\mathrm{~m}$ \\
Peak deflecting voltage at crest phase & 33 & $\mathrm{MV}$ \\
\hline \hline
\end{tabular}

proposed in [4]. Tables I and II show these specifications. These requirements were set before the LCLS was commissioned. Now LCLS operates with normalized emittances as low as $\varepsilon_{n}=0.14 \mu \mathrm{m}$ at $20 \mathrm{pC}$ bunch charge.

Since the temporal resolution of the rf deflector is proportional to $\sqrt{\varepsilon_{n}}$, lower emittance, as well as a higher rf voltage improve the resolution of the deflector to better than $10 \mathrm{fs}$ at $14 \mathrm{GeV}$. Running LCLS for softer x-rays (electron energy 3-4 GeV) allows a temporal resolution of $\sim 2 \mathrm{fs}$.

The layout proposed in [8] utilizes horizontal deflectors placed just after the LCLS undulators, as shown in Fig. 1.

The spent electrons are bent vertically to the main dump and pass through a beam screen that is used to reconstruct the longitudinal phase space of the beam. The screen's vertical coordinate, due to the dispersion of the vertical bend magnet, reveals the electron energy; while the horizontal coordinate, due to the deflector, represents the bunch length coordinate (time). In addition, by switching the FEL on and off, the effects of the FEL on the electron bunch can be time resolved, allowing an absolute measurement of the $\mathrm{x}$-ray pulse length as well as its structure. The FEL process could be switched off by creating a distortion in the beam orbit, see for example [27].

The system uses two 1-meter-long traveling wave rf deflectors powered by one SLAC 50-MW XL-4 klystron. The klystron is capable of producing $1.6 \mu$ s pulses but the deflectors need only about $100 \mathrm{~ns}$. An alternative approach

TABLE II. Approximate specifications for an $X$-band $\mathrm{rf}$ deflecting cavity (Table 3 from [7]).

\begin{tabular}{lcc}
\hline \hline Parameter & Value & Unit \\
\hline Maximum repetition rate & 120 & $\mathrm{~Hz}$ \\
Minimum iris radius & 5 & $\mathrm{~mm}$ \\
$\quad$ (if located after undulator) & & \\
Maximum cavity length (approximate) & 2 & $\mathrm{~m}$ \\
Radio frequency & 11.424 & $\mathrm{GHz}$ \\
Pulse-to-pulse rf phase jitter & 0.05 & $\mathrm{deg}$. \\
Radio-frequency relative & 1 & $\%$ \\
$\quad$ amplitude stability (rms) & & \\
\hline \hline
\end{tabular}




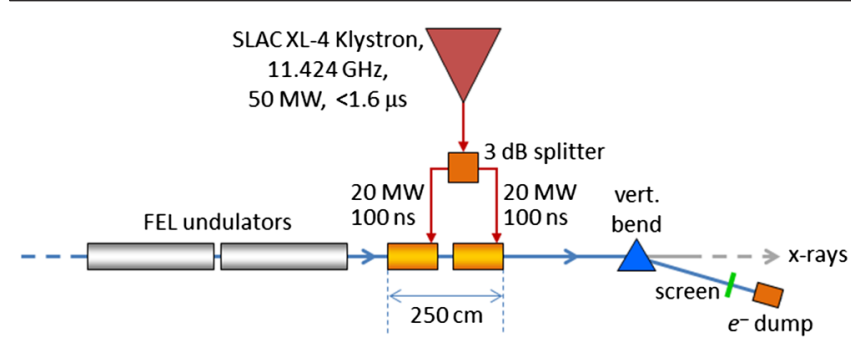

FIG. 1. Schematic layout of LCLS deflector rf system.

of using a single 1.5-meter-long deflector was proposed in [28]. We chose two 1-meter-long structures instead because a shorter defector has a faster fill time, requiring less input power and is easier to braze and handle. Both the shorter fill time and the lower input power reduce the probability of rf breakdown and thus improve reliability during operation.

Further design considerations require a peak electric field of under $150 \mathrm{MV} / \mathrm{m}$, and peak pulse heating below $50{ }^{\circ} \mathrm{C}$. The limits on field and pulse heating are based on results of our rf breakdown testing [13] and pulse heating damage studies [29]. Accelerating structures operating at these field levels have if breakdown probability below $10^{-7}$ per pulse per meter. We found experimentally that the threshold for observable pulse heating damage of the metal surface starts at about $50^{\circ} \mathrm{C}$ [29].

\section{DESIGN APPROACH}

The geometry of the rf deflector is that of a constantimpedance traveling-wave disc-loaded waveguide, similar to early SLAC $S$-band deflectors $[2,3]$. We chose constant impedance to reduce manufacturing cost because the available rf power is sufficient to produce the required transverse kick. The commercial 3D finite-element code HFSS [30] was used for the electrical design.

The aperture of the deflector was set to $10 \mathrm{~mm}$ to stay well clear of the diverging LCLS x-ray beam, especially at long X-ray wavelengths [7]. At this aperture size, with working frequency of $11.424 \mathrm{GHz}$, and practical iris thickness the dipole mode is backward propagating [28]. With rf power and aperture fixed, the kick delivered to the beam weakly depends on phase advance per cell, with a slightly higher value at $\pi / 2$ than at $2 \pi / 3$. We chose structure with $2 \pi / 3$ phase advance per cell as more practical to build and tune since it has fewer cells.

Similar to $X$-band accelerating structures, the deflector is assembled from a set of cells. The cylindrically symmetric structure supports two degenerate dipole modes with two different polarizations. In practical structures these two modes become coupled by imperfections in cell shape and thus will reduce the kick and rotate its direction. To avoid the coupling we introduced rounded polarizing pockets at the outer diameter of the cell. We chose this method instead of holes in the cell iris [16] or polarizing pins to avoid chances of enhancing of fields inside the cell.
A waveguide type coupler $[8,12]$ feeds rf power into the structure. This coupler was developed for $X$-band high gradient accelerating structures but to our knowledge has never been used in rf deflectors. It has several advantages over the traditional, one-sided coupler as in $[2,3,15,20]$. These advantages are no field enhancement in the complicated part of the coupler; no monopole or quadrupole field component that can effect the beam; and the mechanically complicated part of the coupler has looser tolerances than in a traditional coupler. Another feature of this particular realization of the coupler is its extra-long cutoff beam pipe. With these cutoff beam-pipes we eliminated leakage of high power if from the deflector into the LCLS beam pipe where it could degrade the performance of nearby rf beam position monitors. We note that the waveguide coupler occupies more longitudinal space than the traditional coupler, by approximately one cell length. For most applications this length difference is insignificant and well worth the advantages that the waveguide coupler affords.

The parameters of the rf deflector are listed in Table III. We note that the total kick from two deflectors, each powered by $20 \mathrm{MW}$ rf power, is $48 \mathrm{MV}$ and exceeds the original temporal resolution requirement specified in Table I.

\section{A. Cell design}

We chose a traditional disc loaded waveguide with $2 \pi / 3$ phase advance per cell for the cell shape. It has a 2 millimeter-thick rounded iris and two polarizing pockets

TABLE III. Parameters of an $11.424 \mathrm{GHz}$ traveling wave deflector.

\begin{tabular}{lc}
\hline \hline Parameter & Value \\
\hline Frequency & $11.424 \mathrm{GHz}$ \\
Beam pipe diameter & $10 \mathrm{~mm}$ \\
Phase advance per cell & $2 \pi / 3$ \\
Input power & $20 \mathrm{MW}$ \\
Kick per meter in one regular cell at 20 MW & $31 \mathrm{MeV} / \mathrm{m}$ \\
Total number of cells & 117 \\
Total structure kick & $24 \mathrm{MeV}$ \\
Maximum electric field (input coupler) & $100 \mathrm{MV} / \mathrm{m}$ \\
Maximum magnetic field (input coupler) & $405 \mathrm{kA} / \mathrm{m}$ \\
Maximum electric field in first regular cell & $115 \mathrm{MV} / \mathrm{m}$ \\
Maximum magnetic field in first regular cell & $340 \mathrm{kA} / \mathrm{m}$ \\
Peak pulse heating in first regular cell at $110 \mathrm{~ns}$ & $16^{\circ} \mathrm{C}$ \\
Peak pulse heating in coupler at $110 \mathrm{~ns}$ & $23^{\circ} \mathrm{C}$ \\
Total attenuation & $0.62 \mathrm{~Np}$ \\
Power dissipated in the structure & $14 \mathrm{MW}$ \\
Cell quality factor & 6300 \\
Dipole mode frequency separation & $100 \mathrm{MHz}$ \\
Repetition rate & $120 \mathrm{~Hz}$ \\
Group velocity divided by speed of light & $3.2 \%$ \\
Structure length (with beam pipes) & $1.185 \mathrm{~m}$ \\
Fill time & $\sim 110 \mathrm{~ns}$ \\
\hline \hline
\end{tabular}




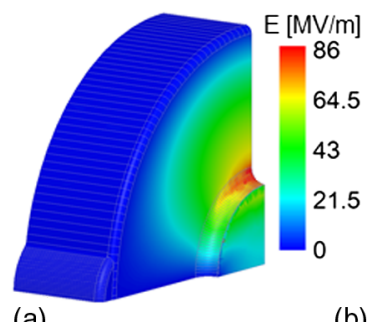

(a)

(b)

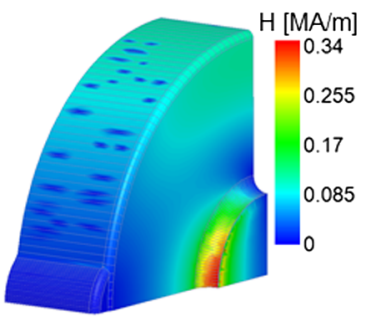

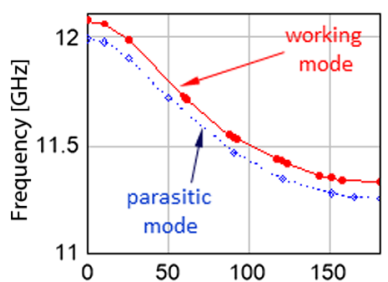

(c) Phase advance per cell [deg.]

FIG. 2. Periodic eigen-solution for regular cell of the rf deflector: (a) surface electric fields; (b) surface magnetic fields; (c) dispersion curves for both working and parasitic modes of orthogonal polarization, with about $100 \mathrm{MHz}$ separation between the curves. Detailed parameters of the cell are listed in Table III.

(a)

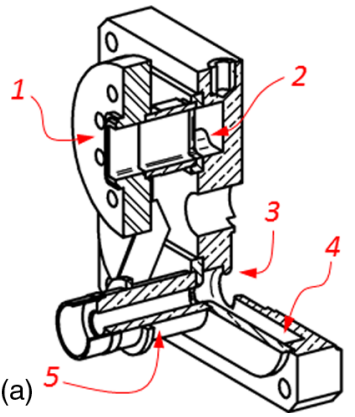

(b)

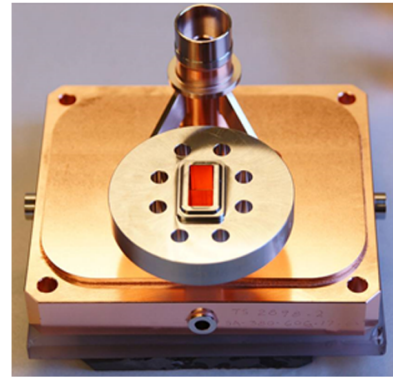

FIG. 3. Input part of the coupler assembly: (a) solid model of the assembly; (b) brazed assembly before joining to disk-loaded waveguide. In (a) are shown 1: input WR90 flange; 2: E-plane splitter; 3: input iris of the first matching cell; 4: H-plane miter bend; 5: extra long beam pipe.

at the outer diameter of the cavity. The shape, the operational surface rf fields, and the dispersion curve for the cell are shown in Fig. 2. We note that the values of peak field and peak pulse heating are significantly below our safe limits.

\section{B. Coupler design}

The rf deflector has identical input and output couplers. Each coupler consists of several parts. Starting from the regular structure cell it has two matching cells; four E-plane WR90 miter bends; E-plane WR90 3dB splitter; and an input-output WR90 waveguide. A sectioned view of the coupler assembly (without matching cells) and the brazed assembly are shown in Fig. 3. The HFSS model of the matching part of the coupler is shown in Fig. 4.

The peak fields and peak pulse heating in the matching cells of the coupler are well below the conservative limit that we set in our design requirements: limit on peak surface electric field of $150 \mathrm{MV} / \mathrm{m}$ vs $115 \mathrm{MV} / \mathrm{m}$ expected in the coupler, and damage limit of $50^{\circ} \mathrm{C}$ vs $23^{\circ} \mathrm{C}$ in the coupler. Since these expected fields are significantly below our requirements we did not expect any measurable breakdown rate. This is now confirmed by many hours of operation of the FACET deflector at $\sim 35 \mathrm{MW}$ of input power without rf breakdowns. We note that the higher surface field in matching cells could be dropped to a level of regular cells as was shown in later developments [31].

The waveguide coupler is symmetric with respect to vertical and horizontal planes as shown in Fig. 4. When excited from both sides by rf fields with opposite phases, it could be modeled as one quarter of the structure with magnetic boundary in the horizontal plane and with electric boundary in the vertical plane. Expansion of the fields into waveguide modes propagating along the deflector axis does not contain either monopole or quadrupole components.

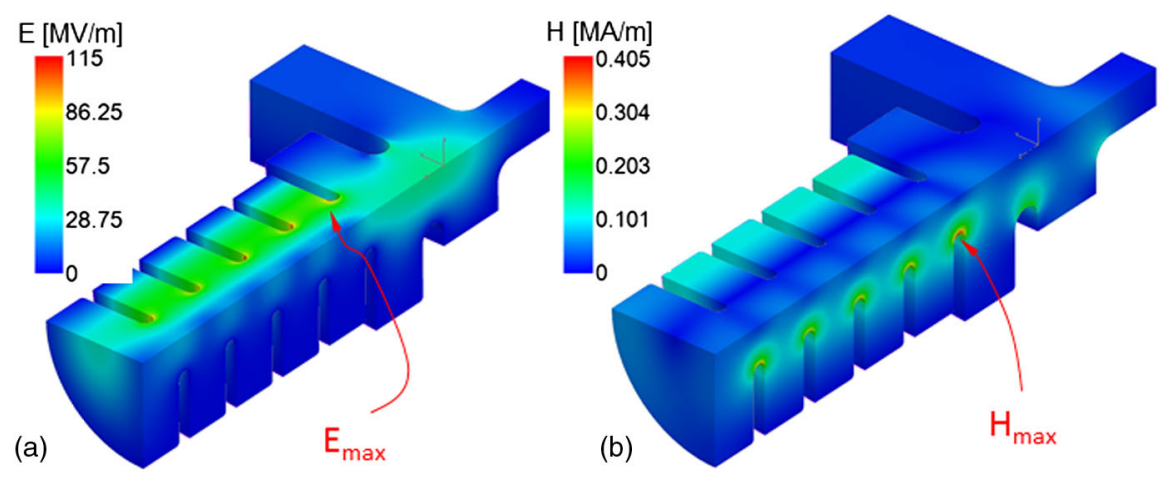

FIG. 4. Waveguide coupler for the rf deflector. Fields are normalized to $20 \mathrm{MW}$ of transmitted power: (a) surface electric fields with maximum value of $100 \mathrm{MV} / \mathrm{m}$; (b) surface magnetic fields with maximum value of $400 \mathrm{kA} / \mathrm{m}$ which corresponds to $23^{\circ} \mathrm{C}$ peak pulse heating for $110 \mathrm{~ns}$ pulse duration. 
(a)

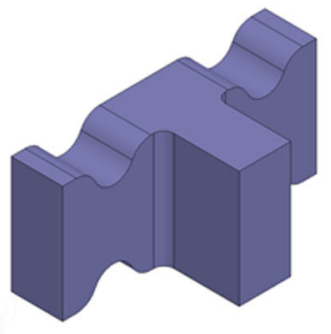

(b)

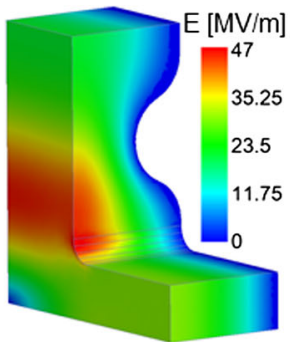

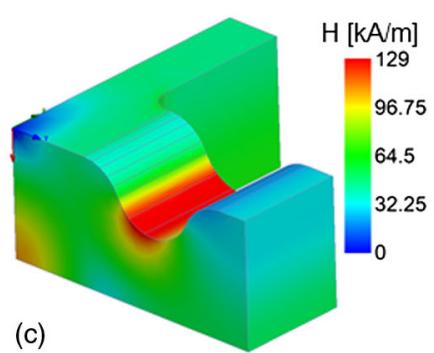

FIG. 5. E-plane splitter for feeding the waveguide coupler of the deflector, fields normalized to $100 \mathrm{MW}$ of transmitted power: (a) solid model of the splitter's rf volume; (b) surface electric fields with maximum value of $47 \mathrm{MV} / \mathrm{m}$; (c) surface magnetic fields with maximum value of $129 \mathrm{kA} / \mathrm{m}$.

(a)

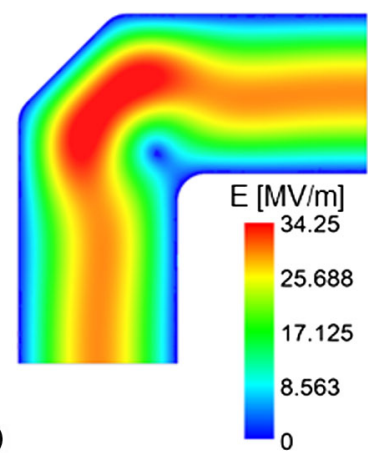

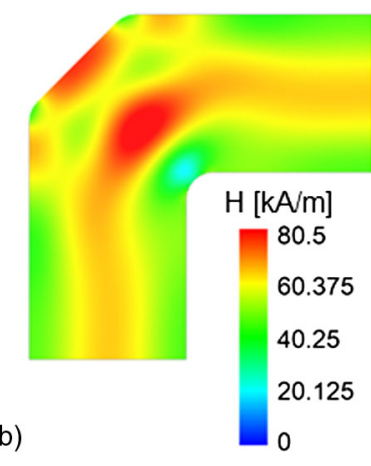

(b)

FIG. 6. H-plane miter bend for feeding the waveguide coupler of the deflector, fields normalized to $100 \mathrm{MW}$ of transmitted power: (a) surface electric fields with maximum value of $34.3 \mathrm{MV} / \mathrm{m}$; (b) surface magnetic fields with maximum value of $80.5 \mathrm{kA} / \mathrm{m}$.

For detailed study of multipole fields in deflector couplers see [9]. We note that quadrupole fields in the couplers could be excited by the beam or by asymmetry of the circuit feeding the waveguide. The discussion about these is outside the scope of this paper.

The coupler is fed from both sides with fields of opposite rf phases. To create such fields we designed the E-plane splitter shown in Fig. 5. The design minimizes both electric and magnetic rf fields to practical limits. The bandwidth of the splitter is $0.27 \mathrm{GHz}$ at $-20 \mathrm{~dB}$.

To transport rf power from the splitter to the structure we used two H-plane miter bends on each side of the structure. Fields in the miter are shown in Fig. 6. The bandwidth of the miter is more than $0.6 \mathrm{GHz}$ at $-50 \mathrm{~dB}$.

\section{MANUFACTURE, TUNING, AND COLD TEST}

The deflector is constructed from two identical halfstructures consisting of 56 regular cells each, two coupler assemblies, two pairs of matching cells, and a single joining ring. The deflector is symmetric around the joining ring. The half-length-structures are diffusion bonded and brazed to the coupler assemblies first, then the entire structure is brazed together along with its water cooling system. All regular cells, matching cells and the joining ring have two symmetrically placed tuning pins for after-braze matching and tuning of the phase velocity. The matching cells and the joining ring do not have polarizing pockets. The deflector structure is stiffened by four stainless steel longitudinal ribs with adjusting screws at midlength to adjust the straightness after brazing. Sockets for alignment tooling balls are brazed into the coupler assemblies.

The whole structure was brazed in several steps. Figure 7 shows all essential components including two diffusion bonded cell stacks, a joining ring, two matching cells, and prebrazed input and output couplers.

The nodal shift method [32] with a reflective plunger was used for tuning and matching of the deflectors. During the measurements and tuning, the deflector was filled with pure dry nitrogen and its temperature was stabilized to $20^{\circ} \mathrm{C} \pm 0.05^{\circ} \mathrm{C}$. Figure 8 shows the measurement setup.

After tuning, the deflector was measured using the nonresonant perturbation technique $[33,34]$. The amplitude and phase of electrical field were obtained by analyzing the reflection from a small glass bead moving along the central axis of the deflector. Figure 9(a) shows the measured electrical field amplitude along the central axis of the deflector. This measurement shows that there is no internal standing wave; this means that the tuning was successful.

Measured after-tuning phase variation between cells of $\pm 2^{\circ}$ is typical for $X$-band accelerating structures. The error comes partially from how precise we could deform the cell

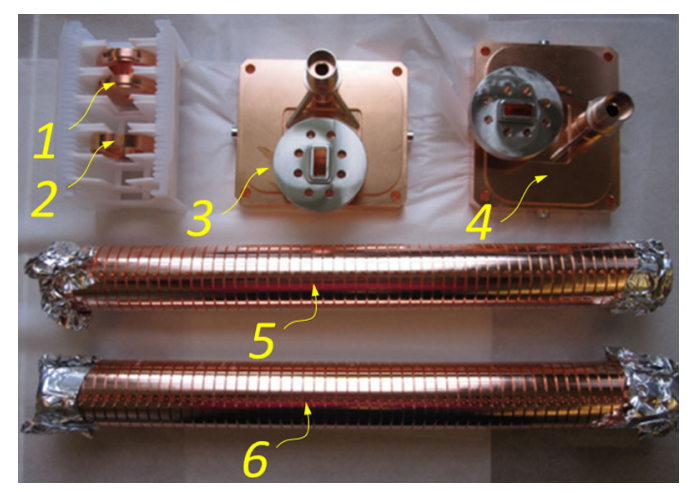

FIG. 7. All components are ready for the final brazing: 1: matching cell; 2 : joining ring; 3,4 : brazed coupler assemblies; 5, 6: diffusion bonded half-length structures. 


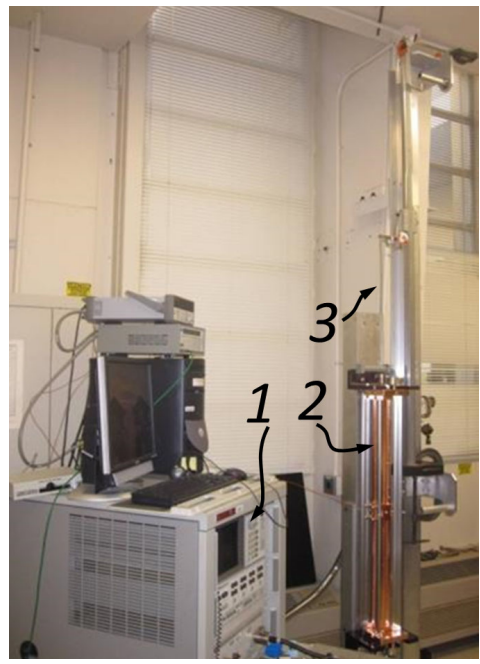

FIG. 8. Setup for microwave measurement and tuning: 1: vector network analyzer; 2: rf deflector; 3: reflective plunger.

and how precise we could measure it. In the case of the deflector, the error is higher because the method for determining the phase of each cell is less accurate. Although rf phase in accelerating and deflecting structures are measured in the same bead-pull configuration, there is a difference. The rf phase of each cell in both cases is measured at the cell's center. In the case of accelerating structures this is a dwell point of the measured phase. In the
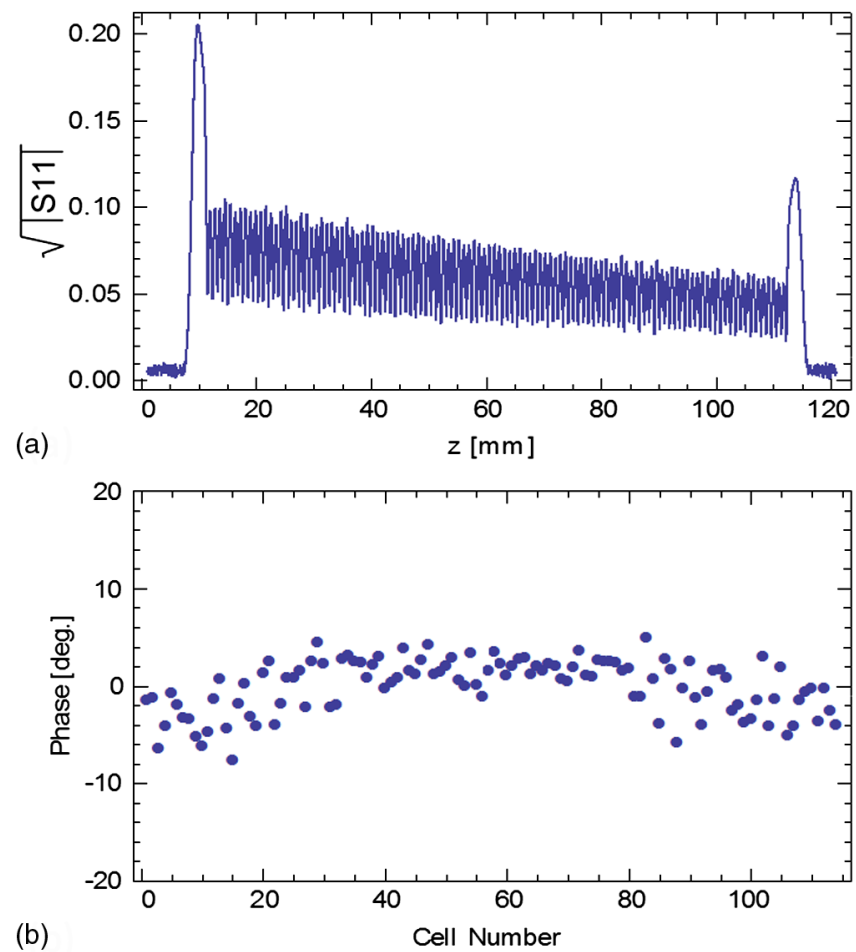

FIG. 9. Results of bead pull measurements: (a) rf electric field amplitude along the central axis of the deflector; (b) difference between beam synchronous phase and rf phase along the central axis of the deflector. case of the deflector the phase there has a steep gradient. The lower accuracy of this phase measurement is one of the reasons why tuning is done by a nodal shift method, not a bead pull, typical for traveling wave accelerating structures.

Unlike in accelerating structures, the deflector on-axis electric fields peaks are located on the centers of the irises (the valleys are located at the centers of cavities). The higher on-axis fields at input and output of the structure are located in the part of the coupler where the eigenmode of the rectangular waveguide is transformed into the hybrid dipole eigenmode of the deflector. Location of this enhanced on-axis electric field is clearly seen in the coupler in Fig. 4. Consistent with simulations, the measured on-axis electric field in the regions of the iris coupling to the input
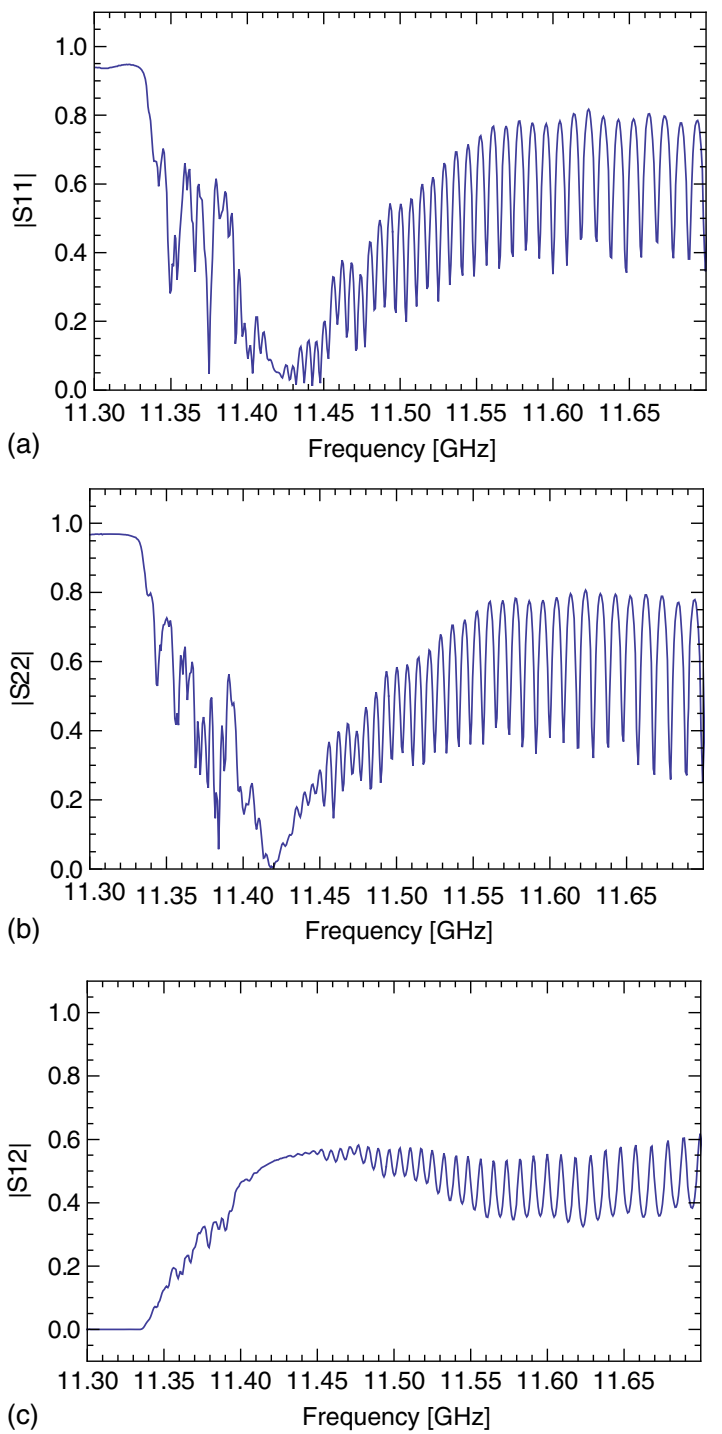

FIG. 10. Measurements of the deflector rf scattering parameters vs frequency: (a) magnitude of the reflection from input coupler with value at operating frequency of 0.03 ; (b) magnitude of the reflection from input coupler with value at operating frequency of 0.04 ; (c) magnitude of the transmission through the deflector with value at operating frequency of 0.53 . 
TABLE IV. Summary of practically achieved parameters from SLAC $X$-band deflector applications.

\begin{tabular}{|c|c|c|c|c|c|}
\hline Parameter & LCLS & FACET & NLCTA & XTA & Unit \\
\hline Beam energy & $4,000-14,000$ & 20,000 & 120 & 75 & $\mathrm{MeV}$ \\
\hline Beam emittance & 0.5 & $\sim 40$ & 2 & 0.55 & $\mu \mathrm{m}$ \\
\hline Structure length (with beam pipes) & $2 \times 1.185$ & 1.185 & 0.432 & 0.293 & $\mathrm{~m}$ \\
\hline Number of regular cells (including joining ring) & $2 \times 113$ & 113 & 27 & 11 & \\
\hline Input power & $17.5+17.5$ & 35 & 20 & 2 & MW \\
\hline On-crest deflecting voltage & 45 & 30 & 6 & 0.9 & MV \\
\hline Resolution achieved & $1-4$ & 70 & 30 & 30 & rms fs \\
\hline Distance to deflector screen & 32 & 14.75 & 3 & 2.5 & $\mathrm{~m}$ \\
\hline Beta functions at rf deflector & 120 at $14 \mathrm{GeV}$ & 150 & 5 & 11 & $\mathrm{~m}$ \\
\hline Beta functions at the screen & 22 at $14 \mathrm{GeV}$ & 0.41 & 8 & 2 & $\mathrm{~m}$ \\
\hline Quadrupole focusing after deflectors & yes & yes & yes & yes & \\
\hline Dipoles after deflectors & yes & yes & no & no & \\
\hline
\end{tabular}

waveguide and first matching cell is a factor of 2 higher than the field near the next iris. On the same iris, both electric and magnetic surface fields are lower than the field in the matching or the regular cells; therefore, we do not expect increased breakdown probability at this location. The contribution of the coupler region to the overall deflection is equivalent to the deflection from about 1.5 regular cells. Figure 9(a) shows the measured rf phase variation of the beam-synchronous phase along the central axis of the deflector vs cell number. Random phase variations of $\pm 5^{\circ}$ has no appreciable effect on deflector operation.

Figure 10 provides the frequency response of the magnitude of the reflection coefficients of the input $\left(S_{11}\right)$ and output $\left(S_{22}\right)$ ends, and the magnitude of transmission through the structure $\left(S_{12}\right)$. The reflection is very small from either side of the deflector. Measured magnitude of the transmission at $11.424 \mathrm{GHz}\left|S_{12}\right|=0.53$ is consistent with the theoretical prediction. This consistency indicates that the assembly and brazing of the structure were performed perfectly. This particular deflector is one of the two installed at LCLS.

\section{APPLICATION EXAMPLES}

We list main parameters of beam diagnostic rf deflector systems in use at SLAC in Table IV. Below are details about these systems.

\section{A. LCLS $X$-band deflector system}

Based on the proposed $X$-band transverse deflector for the LCLS, further studies were made for reconstruction of the X-ray temporal profiles [22]. The start-to-end simulation results were very promising and the LCLS $X$-band transverse deflector project was launched in mid 2011.

To reserve space for future applications directly downstream of the LCLS undulator beam line, the location for the $X$-band deflector was chosen to be at the very end of the LCLS beam line, immediately upstream of the dump bending magnets. There are three quadrupoles between the deflector and the screen. The beam optics has been optimized by relocating one quadrupole magnet from its original position to achieve $90^{\circ}$ betatron phase advance and better resolution. The deflectors, the klystron, the modulator, the waveguide, as well as other supporting devices were designed, fabricated, and tested in 2011 and 2012 $[35,36]$. The two adjacent 1-m-long $X$-band deflecting structures were installed in the beam line in the summer of 2012. Figure 11 shows the deflectors in the LCLS beam line. The entire system was ready for beam commissioning in May 2013.

At present the klystron operates at an output power of about 53 MW. With losses in feeding waveguides, the power at the entrance of each deflector is approximately 17.5 MW. This gives a total maximum deflecting voltage of about $45 \mathrm{MV}$.

An example of a measurement is shown in Fig. 12: (a) shows image on a screen with deflector off; (b) is with deflector on; and (c) shows current beam profiles calculated using measured calibration factors.

The measured rf phase jitter is about $0.15^{\circ} \mathrm{rms}$. Matlabbased graphical user interfaces have been developed to perform the beam-based calibration, the bunch length

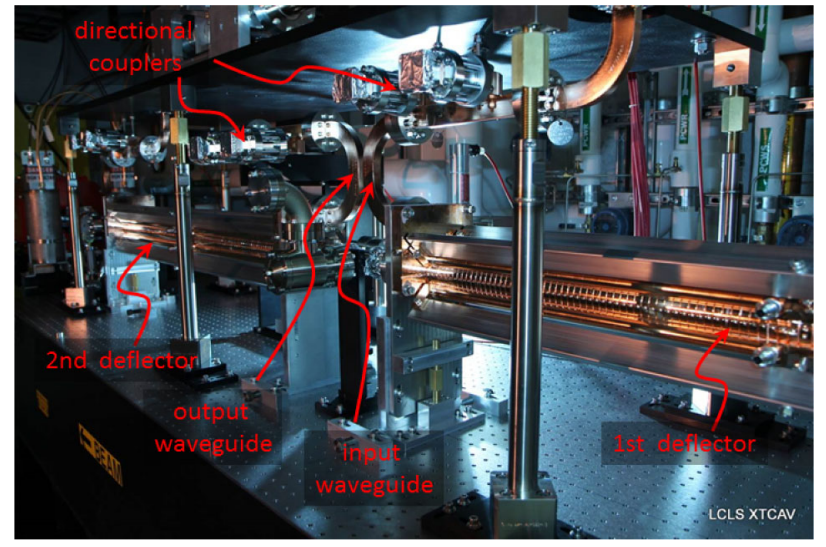

FIG. 11. A photo of the $X$-band rf deflector system installed in the undulator beam line. (Picture credit: P. Krejcik.) 

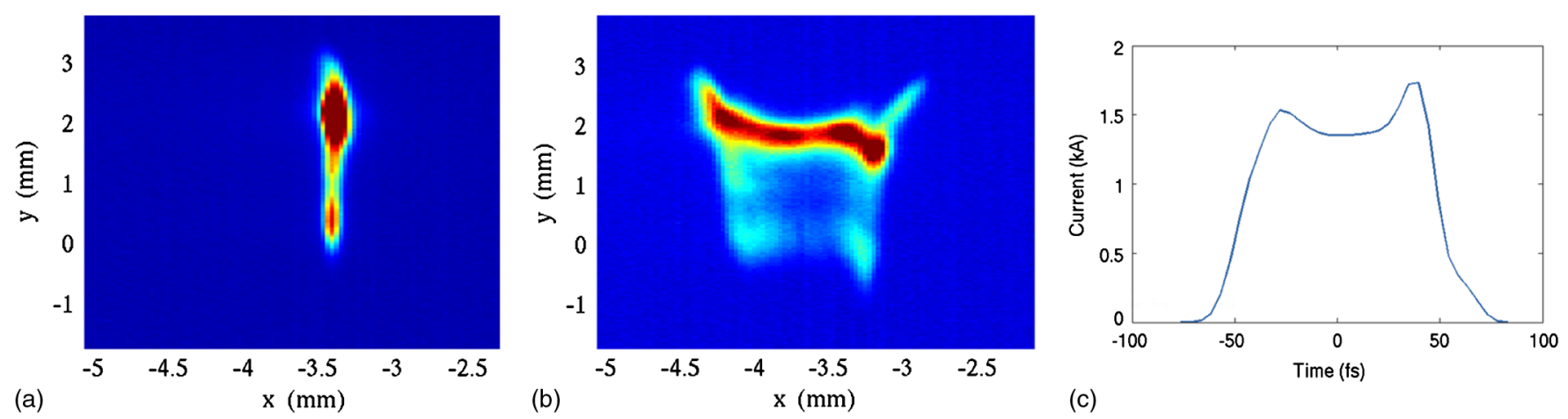

FIG. 12. The measured beam images with deflector (a) off and (b) on at LCLS electron beam energy 13 GeV, bunch charge 150 pC. The curve on (c) shows the current beam profile with absolute units after applying the measured calibration factor.

measurement, and the x-ray temporal profile reconstruction. The klystron amplitude and phase jitters, together with the beam jitters such as arrival time, as well as current and energy jitters, are included in the data analyses. At a beam energy of $4 \mathrm{GeV}$, the preliminary measurement shows a temporal resolution less than $1 \mathrm{fs}$ rms. After further commissioning studies [37], the deflectors are routinely used in tuning and machine studies of LCLS and specifically for single-shot $\mathrm{x}$-ray temporal characterization [38].

\section{B. FACET $X$-band deflector system}

The longitudinal profile of the electron beam at FACET [39] is a crucial characteristic for nearly all experiments at that facility, none more so than the plasma wakefield acceleration (PWFA) experiments, wherein a two-microbunch structure both drives a plasma wake (leading bunch) and rides the accelerating field (trailing bunch) to produce a monoenergetic, high energy beam at the output. When fully compressed, the FACET $20 \mathrm{GeV}$ beam is designed to be as short as $20 \mu \mathrm{m} \mathrm{rms}$ in length. When in the two-microbunch configuration, each microbunch is typically $30 \mu \mathrm{m}$ rms long with a peak-to-peak separation of about $130 \mu \mathrm{m}$. The final energy of the PWFA beam and the interpretation of the results of nearly all experiments at FACET depend highly upon knowledge of the beam's longitudinal profile to at least $20 \mu \mathrm{m}$ resolution [23]. To satisfy this requirement, FACET installed a 1-m-long $X$-band deflector powered by a SLAC XL-4 klystron. Figure 13 shows a photograph of the deflector installed in the FACET beam line.

The rf infrastructure, including the klystron, modulator, and waveguide, was installed, tested, and commissioned over a period of roughly one year, and first put to experimental use in June 2012. The $X$-band deflector was installed in the final arm of the FACET chicane to give a phase advance of $90^{\circ}$ and $180^{\circ}$, respectively, at the Optical Transition Radiation profile monitors located at the beginning of the final focus section of the beam line and at the experimental interaction point. Beam optics configurations were developed based on simulations to optimize the longitudinal resolution of the system while minimizing nonlinearities [23]. A Matlab graphical user interface was used to perform routine deflection calibration and bunch length measurements starting from the summer 2013 run. The longitudinal resolution was estimated to be about $20 \mu \mathrm{m}$. As for now, longitudinal profiles are processed off-line.

We note that the difference in resolution between FACET and LCLS deflector systems is due to several reasons. FACET has higher beam energy, larger emittance, significantly larger bunch charge, a single 1-m-long deflector (instead of two 1-meter-long deflectors as at LCLS), and limitations of optical diagnostics due in part to extremely large beam currents.

\section{NLCTA $X$-band deflectors}

The two $X$-band deflectors installed at SLAC's NLCTA in 2011 have significantly enhanced the capability of this facility [24,25]. One 11-cell deflector is mainly used to increase the beam slice energy spread [25,40], and the other 27-cell deflector is used for measuring the temporal profile of electron beams. These two deflectors are shown in Fig. 14.

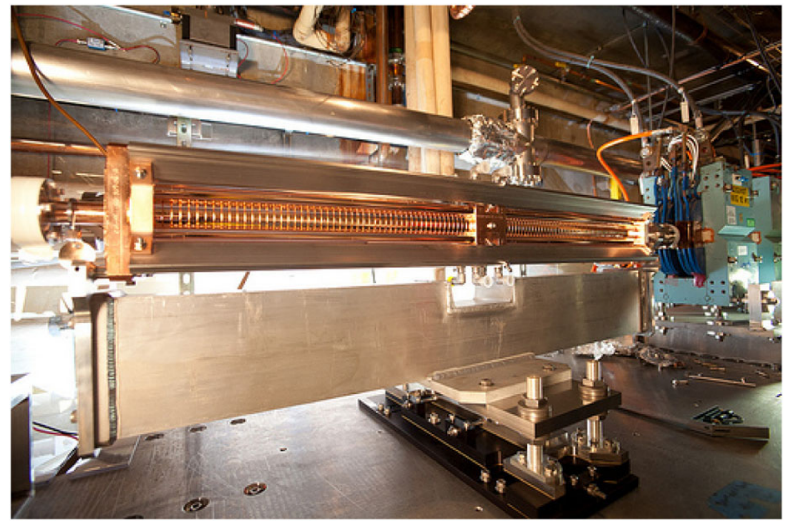

FIG. 13. The 1-m-long $X$-band deflector installed in the FACET beam line. 
(a)

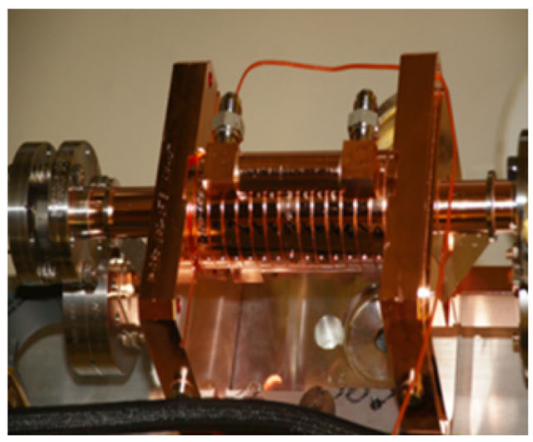

(b)

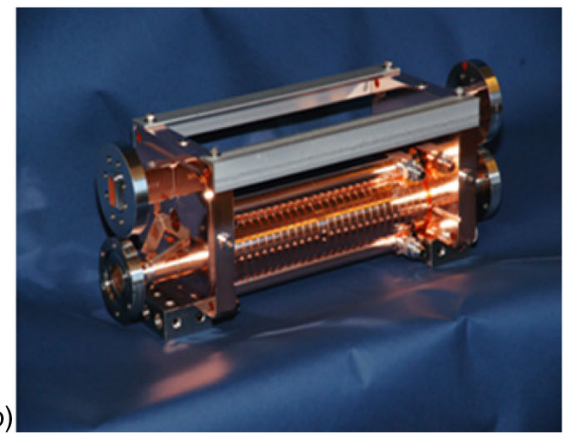

FIG. 14. $X$-band deflectors at SLAC's NLCTA: (a) the 11-cell, and (b) 27-cell.

With $20 \mathrm{MW}$ power (corresponding to about $6 \mathrm{MV}$ kick), the 27-cell deflector provides about 30 fs resolution in the measured beam temporal distribution. Recently, two lasers (one at $800 \mathrm{~nm}$ and the other at $1550 \mathrm{~nm}$ ) have been used to generate periodic density modulation in the electron beam at $12 \mathrm{THz}$ (the difference in frequency of the two lasers). This density modulation has been directly resolved with the 27-cell deflector [41]. The raw image of the streaked electron beam distribution is shown in Fig. 15 where the density modulation at $25 \mu \mathrm{m}$ can be clearly seen.

\section{X-Band Test Area deflector}

The X-Band Test Area (XTA) [42,43] is equipped with an 11-cell deflector shown installed in the beam line in Fig. 16. The construction of the deflector is identical to the NLCTA 11-cell defector shown in Fig. 14(a). It is powered by a 50 MW SLAC XL-4 klystron. However, because of the relatively low electron beam energy, no more than

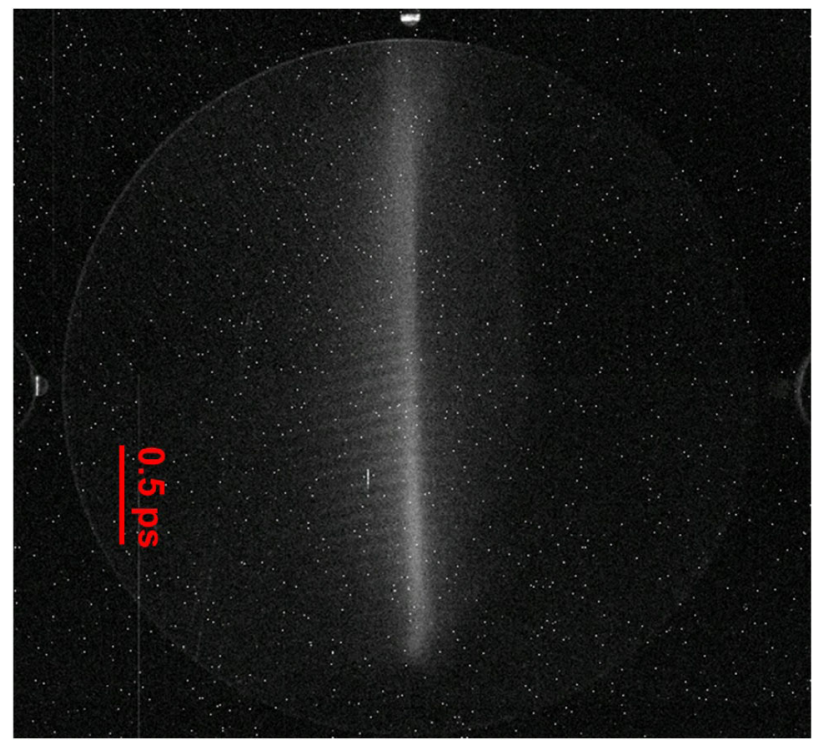

FIG. 15. Raw image of the electron beam streaked by 27-cell $X$-band deflector at SLAC's NLCTA.
2 MW is needed to power the deflector. For beam line parameters shown in Table IV the practical resolution of the system can be as short as $30 \mathrm{fs}$.

During the commissioning of the $X$-band gun the deflector was used to verify the bunch length. Despite strong rf phase jitter in the main rf power source, an accurate determination of the bunch length was possible by running a large number of samples. An example of such a measurement is shown in Fig. 17. The bunch length was measured to be $476 \mathrm{fs}$ rms at $290 \mathrm{pC}$ bunch charge and $70 \mathrm{MeV}$, confirming predictions from simulations. Continued operation of the deflector is planned at XTA to measure slice emittances and thus fully characterize the beam.

Ultrafast electron diffraction experiments are planned at the XTA. As part of this program the deflector will be used to image dynamic properties of crystals in phase transition. This approach was presented in [44] as an improvement on the earlier work [45]. Note that the resolution of the $S$-band deflector in [45] was limited to $200 \mathrm{fs}$. With the XTA detector the resolution is anticipated to be $\sim 5 \mathrm{fs}$ at $5 \mathrm{MeV}$. This improvement in resolution comes from shorter $X$-band wavelength, larger maximum kick, choice of beta function, and from the smaller transverse emittance of the XTA bunch.

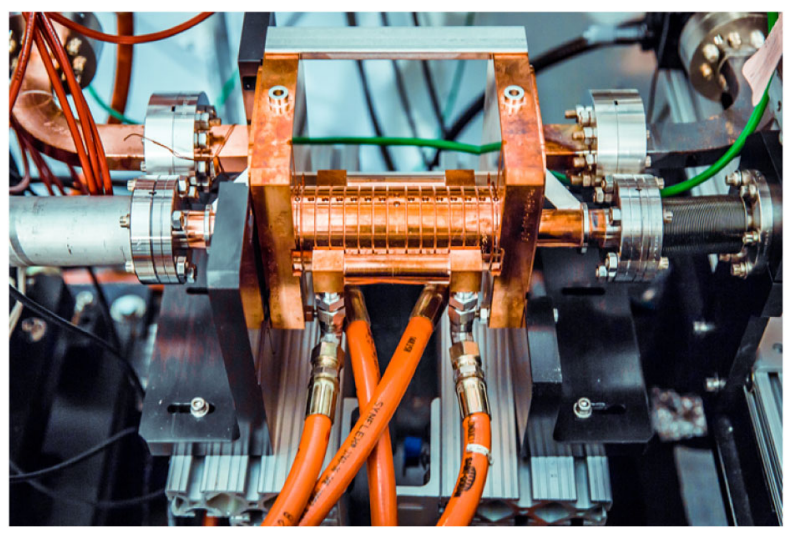

FIG. 16. 11-cell deflector installed in X-Band Test Area. 


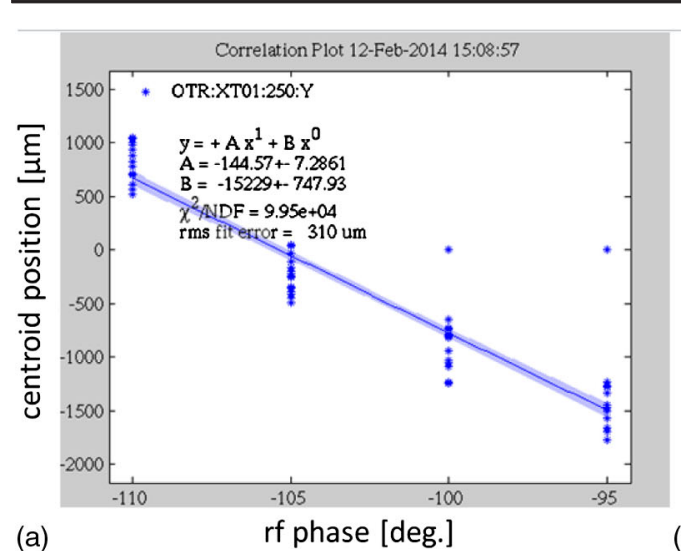

(a)

rf phase [deg.]

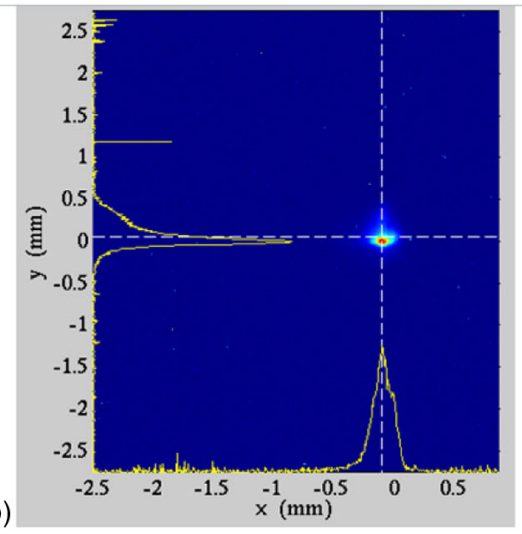

(b)

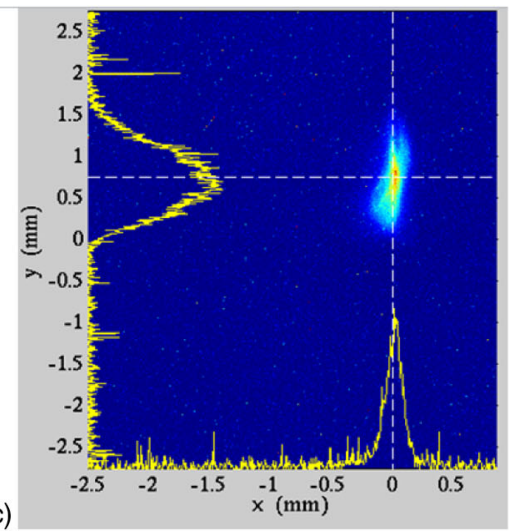

FIG. 17. Example of bunch measurements using XTA $X$-band detector: (a) calibration curve, transverse bunch centroid position-to-rf phase; (b) rf deflector off; (c) vertically streaked bunch profile observed on a Yttrium Aluminum Garnet screen.

\section{SUMMARY AND DISCUSSION}

Motivated by LCLS requirements, we designed, built, and commissioned a set of high frequency $X$-band deflectors to measure beam longitudinal space charge distribution and energy spread to better than 10 fs resolution at full LCLS energy (14 GeV), and with 70 fs resolution at full FACET energy $(20 \mathrm{GeV})$. The high frequency and high gradient features allow these devices to reach unprecedented performances.

A total of seven $X$-band deflectors of this type have been built at SLAC. Six are successfully operating at SLAC and one was shipped to KEK for use at the B-factory injector. This project successfully capitalized on the U.S. Department of Energy's (DOE) investment in $X$-band high gradient technology. As a result, the FACET deflector operates at up to $35 \mathrm{MW}$ input power and thus far has not exhibited any measurable breakdown rate.

We envision that in the future these deflectors could be used in much more powerful systems such as four 1-m-long deflectors fed by one XL-4 klystron with rf pulse compressor to give the beam a 92 MV kick [8].

\section{ACKNOWLEDGMENTS}

This work was supported by the U.S. Department of Energy Contract No. DE-AC02-76SF00515. The achievement of the SLAC $X$-band deflector systems has been enabled by decades of development of $X$-band high gradient technology at SLAC which was supported by the Next-Linear Collider program and recently by the US High Gradient Collaboration whose spokesperson is Sami G. Tantawi. We thank R. Reed for the manufacturing drawing set of the original deflector. We thank $\mathrm{Z}$. Li for his simulations which help in understanding deflector measurements. We thank B. McKee for the installation, C. Adolphsen, S. Chu, and F. Wang for designing, instrumenting and operating of the NLCTA rf stations.
[1] Y. Ding, A. Brachmann, F.-J. Decker, D. Dowell, P. Emma, J. Frisch, S. Gilevich, G. Hays, P. Hering, Z. Huang, R. Iverson, H. Loos, A. Miahnahri, H.-D. Nuhn, D. Ratner, J. Turner, J. Welch, W. White, and J. Wu, Phys. Rev. Lett. 102, 254801 (2009).

[2] O. H. Altenmueller, R. R. Larsen, and G. Loew, Rev. Sci. Instrum. 35, 438 (1964).

[3] G. Loew and O. Altenmueller, Report No. SLAC-PUB135, 551, 1965.

[4] P. Emma, J. Frisch, and P. Krejcik, Reports No. LCLS-TN00-12 and No. SLAC-PUB-8864, 2000.

[5] R. Akre, L. Bentson, P. Emma, and P. Krejcik, in Proceedings of the 19th IEEE Particle Accelerator Conference PAC2001, Chicago, IL, 2001 (IEEE, Piscataway, NJ, 2001), pp. 2353-2355.

[6] P. Emma, Nat. Photonics 4, 641 (2010).

[7] P. Emma, LCLS Technical Note, 2006.

[8] V. Dolgashev, Presented at 13th Advanced Accelerator Concepts Workshop, Santa Cruz, California, USA, 2008, Report No. SLAC-WP-084; http://slac.stanford.edu/pubs/ slacwps/wp07/slac-wp-084.pdf.

[9] P. K. Ambattu, G. Burt, A. Grudiev, V. Dolgashev, and A. Dexter, Phys. Rev. ST Accel. Beams 16, 062001 (2013).

[10] V. A. Dolgashev and J. Wang, in Proceedings of the 15th Advanced Accelerator Concepts Workshop (AAC 2012), Austin, Texas, USA, 2012 [AIP Conf. Proc. 1507, 682 (2012)].

[11] J. Wang, Presented at X-Band Structures and Beam Dynamics Workshop, Cockcroft Institute, UK, 2008, Report No. SLAC-PUB-13554; http://www.slac.stanford .edu/cgi-wrap/getdoc/slac-pub-13554.pdf.

[12] C. Nantista, S. Tantawi, and V. Dolgashev, Phys. Rev. ST Accel. Beams 7, 072001 (2004).

[13] V. Dolgashev, S. Tantawi, Y. Higashi, and B. Spataro, Appl. Phys. Lett. 97, 171501 (2010).

[14] V. Dolgashev, Proceedings of ICFA Beam Dynamics MiniWorkshop on Deflecting/Crabbing Cavity Applications in Accelerators, Cockcroft Institute, UK, 2010 [http://indico .cern.ch/conferenceDisplay.py?confId=83532].

[15] A. Murokh, R. Agustsson, S. Boucher, P. Frigola, D. Alesini et al., in Proceedings of the 11th European Particle 
Accelerator Conference, Genoa, Italy, 2008 (EPS-AG, Geneva, 2008), pp. 1215-1217.

[16] L. Faillace, R. Agustsson, P. Frigola, A. Murokh, J. Rosenzweig, D. Alesini, and V. Yakimenko, in Proceedings of IPAC2010 (Kyoto, Japan, 2010), pp. 1206-1208.

[17] R. Agustsson, L. Faillace, A. Murokh, S. Storms, J. Rosenzweig, D. Alesini, V. Dolgeshev, and V. Yakimenko, in Proceedings of the 3rd International Particle Accelerator Conference, New Orleans, LA, 2012 (IEEE, Piscataway, NJ, 2012) pp. 3389-3391.

[18] J. Haimson, B. Mecklenburg, G. Stowell, and B. Ishii, AIP Conf. Proc. 647, 810 (2002).

[19] J. Haimson, AIP Conf. Proc. 737, 95 (2004).

[20] R. England, B. O'Shea, J. Rosenzweig, G. Travish, and D. Alesini, in Proceedings of the 21 st Particle Accelerator Conference, PAC-2005, Knoxville, TN, 2005 (IEEE, Piscataway, NJ, 2005), pp. 2627-2629.

[21] A. Dexter, G. Burt, P. Ambattu, V. Dolgashev, and R. Jones, Nucl. Instrum. Methods Phys. Res., Sect. A 657, 45 (2011).

[22] Y. Ding, C. Behrens, P. Emma, J. Frisch, Z. Huang, H. Loos, P. Krejcik, and M.-H. Wang, Phys. Rev. ST Accel. Beams 14, 120701 (2011).

[23] M. Litos, M. Bionta, V. Dolgashev, R. England, D. Fritz, S. Gilevich, P. Hering, and M. Hogan, in Proceedings of the 24th Particle Accelerator Conference, PAC-2011, New York, 2011 (IEEE, New York, 2011), pp. $568-570$.

[24] D. Xiang, E. Colby, M. Dunning, S. Gilevich, C. Hast, K. Jobe, D. McCormick, J. Nelson, T. O. Raubenheimer, K. Soong, G. Stupakov, Z. Szalata, D. Walz, S. Weathersby, and M. Woodley, Phys. Rev. ST Accel. Beams 14, 112801 (2011).

[25] D. Xiang, E. Colby, M. Dunning, S. Gilevich, C. Hast, K. Jobe, D. McCormick, J. Nelson, T. O. Raubenheimer, K. Soong, G. Stupakov, Z. Szalata, D. Walz, S. Weathersby, and M. Woodley, Phys. Rev. Lett. 108, 024802 (2012).

[26] C. Limborg-Deprey, C. Adolphsen, T. Chu, M. Dunning, R. Jobe et al., in Proceedings of the 24th Particle Accelerator Conference, PAC-2011, New York, 2011 (IEEE, New York, 2011), pp. 133-135.

[27] D. Ratner et al., in Proceedings of 31st International Free Electron Laser Conference, FEL2009, Liverpool, UK, 2009, pp. 221-224; http://epaper.kek.jp/FEL2009/.

[28] J. Wang and S. Tantawi, in Proceedings of of the 24th Linear Accelerator Conference, LINAC2008, Victoria, British Columbia, Canada, 2008, pp. 966-968; http://accelconf.web.cern.ch/Accelconf/LINAC08/index .htm.

[29] L. Laurent, S. Tantawi, V. Dolgashev, C. Nantista, Y. Higashi, M. Aicheler, S. Heikkinen, and W. Wuensch, Phys. Rev. ST Accel. Beams 14, 041001 (2011).

[30] www.ansys.com.

[31] G. Burt, P. Ambattu, A. Dexter, T. Abram, V. Dolgashev et al., arXiv:0903.2116.
[32] E. L. Ginzton, Microwave Measurements (McGraw-Hill, New York, 1957).

[33] C. W. Steele, IEEE Trans. Microwave Theory Tech. 14, 70 (1966).

[34] K. B. Mallory and R. H. Miller, IEEE Trans. Microwave Theory Tech. 14, 99 (1966).

[35] P. Krejcik, Y. Ding, J. Frisch, Z. Huang, H. Loos, J. Wang, M.-H. Wang, C. Behrens, and P. Emma, in Proceedings of International Beam Instrumentation Conference, IBIC2012, Tsukuba, Japan, 2012; http://ibic12.kek.jp/prepress/index .htm.

[36] Y. Ding, J. Frisch, Z. Huang, P. Krejcik, J. R. Lewandowski, H. Loos, J. W. Wang, M.-H. Wang, J. Welch, and C. Behrens, in Proceedings of the 4th International Particle Accelerator Conference, IPAC-2013, Shanghai, China, 2013 (JACoW, Shanghai, China, 2013), pp. 2091-2093.

[37] P. Krejcik, F.-J. Decker, Y. Ding, J. Frisch, Z. Huang, J. Lewandowski, H. Loos, J. Turner, J. Wang, M.-H. Wang, and J. Welch, in Proceedings of 2nd International Beam Instrumentation Conference, IBIC2013, Oxford, UK, 2013, pp. 308-311; http://accelconf.web.cern.ch/ AccelConf/IBIC2013/.

[38] C. Behrens, F.-J. Decker, Y. Ding, V. A. Dolgashev, J. Frisch, Z. Huang, P. Krejcik, H. Loos, A. Lutman, T. J. Maxwell, J. Turner, J. Wang, M.-H. Wang, J. Welch, and J. Wu, Nat. Commun. 5, 3762 (2014).

[39] C. Clarke, E. Adli, S. Corde, F. Decker, R. England et al., in Proceedings of Proceedings of the 26th International Linear Accelerator Conference, LINAC2012, Tel-Aviv, Israel, 2012, pp. 802-806; http://accelconf.web.cern.ch/ AccelConf/LINAC2012/index.htm.

[40] C. Behrens, Z. Huang, and D. Xiang, Phys. Rev. ST Accel. Beams 15, 022802 (2012).

[41] M. Dunning, C. Hast, E. Hemsing, K. Jobe, D. McCormick, J. Nelson, T. O. Raubenheimer, K. Soong, Z. Szalata, D. Walz, S. Weathersby, and D. Xiang, Phys. Rev. Lett. 109, 074801 (2012).

[42] C. Limborg-Deprey, C. Adolphsen, M. Dunning, S. Gilevich, C. Hast, R. Jobe, D. McCormick, A. Miahnahri, T. Raubenheimer, A. Vlieks, D. Walz, and S. Weathersby, in Proceedings of Proceedings of the 26th International Linear Accelerator Conference, LINAC2012, Tel-Aviv, Israel, 2012, pp. 234-236; http://accelconf.web.cern.ch/ AccelConf/LINAC2012/index.htm.

[43] C. Limborg et al., Presented at ICFA-Workshop on Physics and Applications of High Brightness Beams, HBEB2013, Puerto Rico, US, 2013; http://pbpl.physics.ucla.edu/ HBEB2013/Program.html.

[44] C. Limborg et al., in Presented at Workshop on Ultrafast Electron Sources for Diffraction and Microscopy Applications, UESDM2012, Los Angeles, CA, 2012; http://pbpl .physics.ucla.edu/UESDM_2012/Program.html.

[45] R. Li, W. Huang, Y. Du, L. Yan, Q. Du, J. Shi, J. Hua, H. Chen, T. Du, H. Xu, and C. Tang, Rev. Sci. Instrum. 81, 036110 (2010). 